\title{
Partial Maxillectomy
}

National Cancer Institute

\section{Source}

National Cancer Institute. Partial Maxillectomy. NCI Thesaurus. Code C51657.

Surgery to remove part of the maxilla. 